\title{
DaTQUANT: The Future of Diagnosing Parkinson Disease
}

\author{
Jacquelyn E. Brogley \\ Gateway Community College, Nuclear Medicine Technology Program, Phoenix, Arizona
}

CE credit: For CE credit, you can access the test for this article, as well as additional JNMT CE tests, online at https://www.snmmilearningcenter.org. Complete the test online no later than March 2022. Your online test will be scored immediately. You may make 3 attempts to pass the test and must answer $80 \%$ of the questions correctly to receive $1.0 \mathrm{CEH}$ (Continuing Education Hour) credit. SNMMI members will have their CEH credit added to their VOICE transcript automatically; nonmembers will be able to print out a CE certificate upon successfully completing the test. The online test is free to SNMMI members; nonmembers must pay $\$ 15.00$ by credit card when logging onto the website to take the test.

Idiopathic Parkinson disease (PD) is a progressive neurologic condition that affects the dopamine transporters in the substantia nigra of the brain. Currently, more than 10 million people are living with this disease worldwide, with thousands of newly diagnosed and undiagnosed cases added every year. The disease is difficult to differentiate from other similar disorders, as symptoms widely vary and can mimic other conditions. Classic PD symptoms may look similar to essential tremor and other parkinsonian syndromes. The ${ }^{123}$-ioflupane dopamine transporter (DaT) protocol differentiates PD from essential tremor through in vivo testing with SPECT imaging. The DaT protocol commonly relies on a semiquantitative analysis and visual interpretations of the images, which may produce inaccurate results due to human error. DaTQUANT software (GE Healthcare) was created in 2013 as an adjunct processing tool with advanced quantitative uptake methods and a designated normals database for a more accurate assessment of a patient's case. DaTQUANT has proven to be a vital protocol component for an accurate differentiation of PD from essential tremor. Current use of the software has been rather limited, so a greater push for education and implementation will be key for its success.

Key Words: Parkinson's disease; ${ }^{123}$ I loflupane; dopamine transporter; DaTQUANT®

J Nucl Med Technol 2019; 47:21-26

DOI: 10.2967/jnmt.118.222349

\section{A} s many as 1 million Americans live with Parkinson disease, which is more than the combined number of people diagnosed with multiple sclerosis, muscular dystrophy, and Lou Gehrig's disease. Approximately 60,000 Americans are diagnosed with Parkinson disease each year, and this number does not reflect the thousands of cases that go undetected (1). In correlation to the 10 million cases worldwide, this statistic brings attention to the critical issue of diagnosing and managing the disease. Presently, there are no definitive blood tests

Received Oct. 28, 2018; revision accepted Nov. 26, 2018.

For correspondence or reprints contact: Jacquelyn E. Brogley, Gateway Community College, 108 40th St., Phoenix, AZ 85034.

E-mail: jacbroges@gmail.com

Published online Jan. 25, 2019.

COPYRIGHT @ 2019 by the Society of Nuclear Medicine and Molecular Imaging. or imaging modalities that can diagnose Parkinson disease per se, as the cause remains largely unknown and symptoms widely vary. In trying to understand what Parkinson disease is, researchers have discovered the origins of symptom causation within the substantia nigra region of the brain. This information has been primarily gained through postmortem autopsies of patients who lived with symptoms related to parkinsonian syndromes. Existing diagnostic practices need to be improved to better treat those living with the disease before it is too late. This continuing education article will provide an overview of Parkinson disease, current diagnostic practices, the ${ }^{123} \mathrm{I}$-ioflupane dopamine transporter scan, and the processing tool known as DaTQUANT. The protocol has been shown to effectively rule out Parkinson disease from essential tremor, providing a patient with more information toward a diagnosis and symptom management.

\section{PARKINSON DISEASE}

Parkinson disease is categorized as a paralysis agitans, or disorder of movement control due the degeneration of neurons within the substantia nigra (2). Located in the ventral midbrain, the substantia nigra is lined with an extensive network of axonal processes that innervate the basal ganglia (2). The basal ganglia regulates motor control, together with a wider variety of roles such as motor learning, executive functions, behavior, and emotions (2). The substantia nigra is a distinct region within the basal ganglia, consisting of the substantia nigra pars compacta and the substantia nigra pars reticulata (2). Both sections are characterized by a blackened appearance from a dark polymer pigment known as neuromelanin (Figs. 1A and 1B) (2). The dopamine transporter neurons reside in the substantia nigra pars compacta, whereas $\gamma$-aminobutyric acid neurons fill the pars reticulata portion. The substantia nigra region communicates with the caudate nucleus and putamen regions of the basal ganglia through the release of dopamine, the neurotransmitter responsible for an organism's fluid movements. Parkinson disease manifests as the deterioration of the substantia nigra dopamine neurons, which ultimately slows the release of dopamine needed for seamless movement. A patient experiencing parkinsonian-related symptoms may notice a slight resting tremor, rigidity, bradykinesia, hypokinesia, 


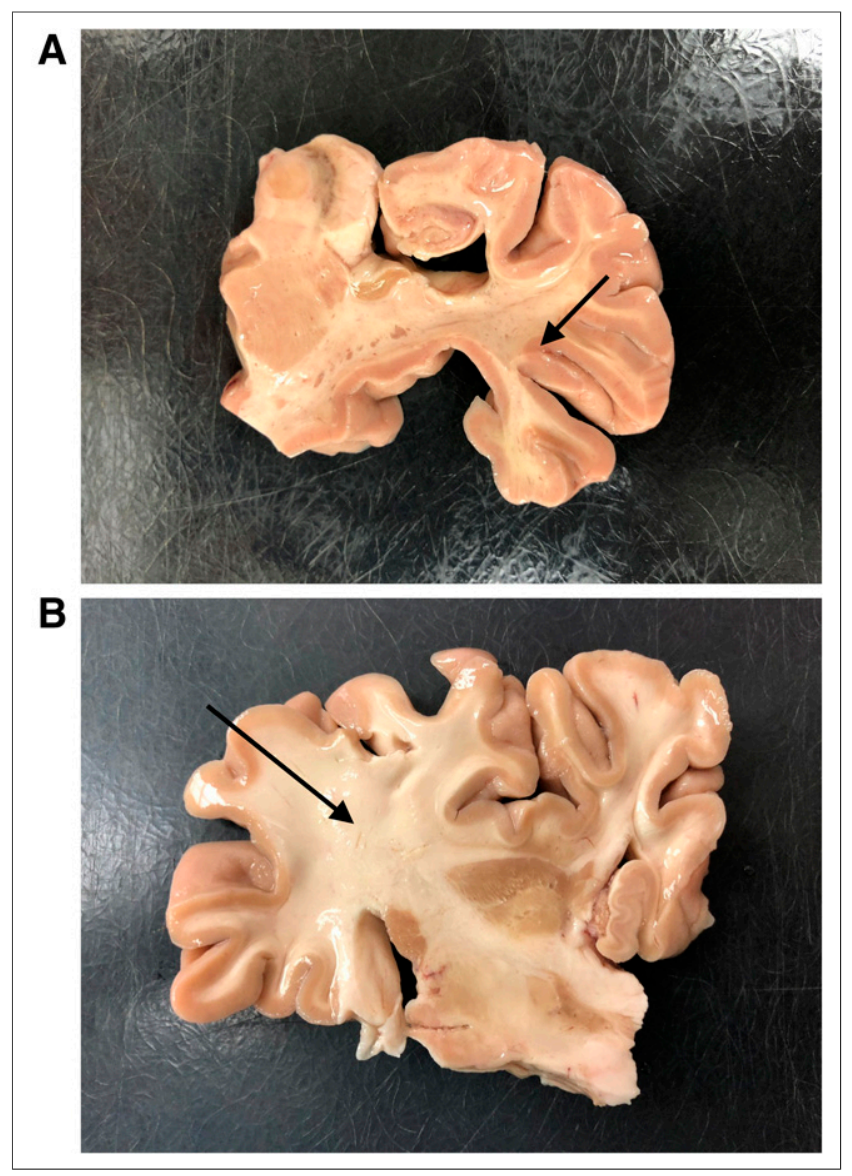

FIGURE 1. (A) Control sample of healthy brain tissue of substantia nigra. Darkened areas (arrow demonstrating portion of darkened outline) show pigmented neuromelanin portions of substantia nigra pars compacta. (B) Positive Parkinson disease tissue sample. Lightened areas demonstrate lack of neuromelanin. (Reprinted with permission of the Tissue User Note, Banner Sun Health Research Institute.)

and loss of postural reflexes (2). These cardinal symptoms are common to other clinical parkinsonian syndromes such as progressive supranuclear palsy, multiple-system atrophy, vascular parkinsonism, corticobasal ganglionic degeneration, and dementia with Lewy bodies (2). These disorders are categorized as parkinsonian syndromes due to the relative symptoms experienced, which may appear as idiopathic Parkinson disease. This makes for a difficult diagnosis and ultimately symptom management, as several of these are considered drug-resistant parkinsonian syndromes. The drug commonly prescribed for symptom management is a chemical precursor of dopamine known as levodopa, which is combined with the enzyme inhibitor carbidopa (3). The medication is a natural chemical that crosses the blood-brain barrier and is converted to dopamine to replenish the low or nonexistent levels present. Levodopa is combined with carbidopa, which protects levodopa from early conversion to dopamine outside the brain, which prevents or lessens side effects such as nausea (4). There are several symptom treatment options for Parkinson disease, but levodopa has been shown to be most effica- cious in managing day-to-day symptoms experienced by the patient.

The timeline of disease indication to a definitive diagnosis can range from 2 mo to $18 \mathrm{y}$ depending on the clinical presentation (5). According to $\mathrm{Ba}$ and Martin, “....misdiagnosis of conditions such as essential tremor, vascular parkinsonism, multiple system atrophy, and progressive supranuclear palsy implies that 5-25\% of patients with an initial diagnosis of Parkinson disease may be inappropriately managed" (5). This issue leads the discussion to what is currently practiced for an accurate evaluation of Parkinson disease from the other common syndromes.

\section{CURRENT DIAGNOSTIC PRACTICES}

Neurologists have developed a method for initial assessment and monitoring of Parkinson disease progression through a symptom-based diagnosis. The technique includes a timeline of physical and neurologic symptoms in conjunction with a medical history and existing physical capabilities or limitations. This has been the standard of diagnosis since the 1950s when Parkinson disease was delineated from other neurologically debilitating conditions across the spectrum of diseases (6).

Another tool used is the Unified Parkinson Disease Rating scale, which is the most well-established scale for assessing patient disability and impairment (7). This evaluative tool was created by the Movement Disorder Society Task Force of the International Parkinson and Movement Disorder Society (7). The scale was formulated with a quantification of symptom severity, numbering zero as normal to 4 as the most acute disease symptoms experienced (7). The results of scale may be included during the initial clinical assessment of a patient's symptoms.

Other potentially useful diagnostic imaging studies include high-field-strength (1.5 T) heavily T2-weighted MRI, ${ }^{18} \mathrm{~F}$-fluorodopa PET, ${ }^{11} \mathrm{C}$-raclopride imaging of dopamine D2 receptors, and brain parenchyma sonography (7). Although these neuroimaging techniques are promising, further refinement in resolution and improvement in sensitivity are needed before their diagnostic potential is fully realized (7).

\section{3|-IOFLUPANE MECHANISM OF ACTION}

In current practice, the ${ }^{123}$ I-ioflupane dopamine transporter (DaT) protocol is the only diagnostic imaging modality approved by the U.S. Food and Drug Administration for the evaluation of Parkinson disease (8). On January 14, 2011, GE Healthcare announced the approval of the ${ }^{123}$ Iioflupane injection: "the DaT scan Ioflupane ${ }^{123}$ I injection is a radiopharmaceutical indicated for striatal dopamine transporter visualization using SPECT brain imaging to assist in the evaluation of adult patients with suspected Parkinsonian syndromes. In these patients, DaT scans may be used to help differentiate essential tremor from tremor due to idiopathic Parkinson disease, multiple system atrophy and progressive supranuclear palsy. The DaT scan is an adjunct to other diagnostic evaluations" (8).

${ }^{123}$ I-ioflupane is used to demonstrate the location and concentration of dopamine transporters in the synapses (9). 
Dopamine transporters refer the transmembrane proteins in the presynaptic membrane of the dopaminergic synapse that transport dopamine from the synaptic cleft to the postsynaptic dopamine receptors (Fig. 2). They can also transport dopamine back into the nigrostriatal nerve terminals for either storage or degradation (9). The ${ }^{123}$ I-ioflupane tracer specifically tags to this component of the nigrostriatal dopaminergic pathway located on the presynaptic nerve terminals to determine the integrity of the system's functionality in releasing dopamine.

According to the Society of Nuclear Medicine and Molecular Imaging (SNMMI) practice guideline for dopamine transporter imaging, "DaT concentrations are lower in presynaptic Parkinsonian syndromes, which include Parkinson disease, multiple system atrophy, and progressive supranuclear palsy, and are also lower in dementia with Lewy bodies" (9). Parkinsonian syndromes such as essential tremor, drug-induced parkinsonism, and psychogenic parkinsonism will have relatively normal presynaptic dopaminergic loss. In differentiating the levels of dopaminergic loss, the tracer can demonstrate a significant variance between Parkinson disease from essential tremor. However, the protocol cannot discriminate idiopathic Parkinson disease from multiplesystem atrophy, progressive supranuclear palsy, dementia with Lewy bodies, or any other parkinsonian syndromes so far mentioned. Despite this limitation, a DaT scan can adequately provide a patient with an accurate in vivo assessment at the striatal level.

\section{DAT PROTOCOL}

The DaT scan protocol begins with obtaining the patient's clinical history and contraindications, followed by the administration of a thyroid-blocking agent such as Lugol's

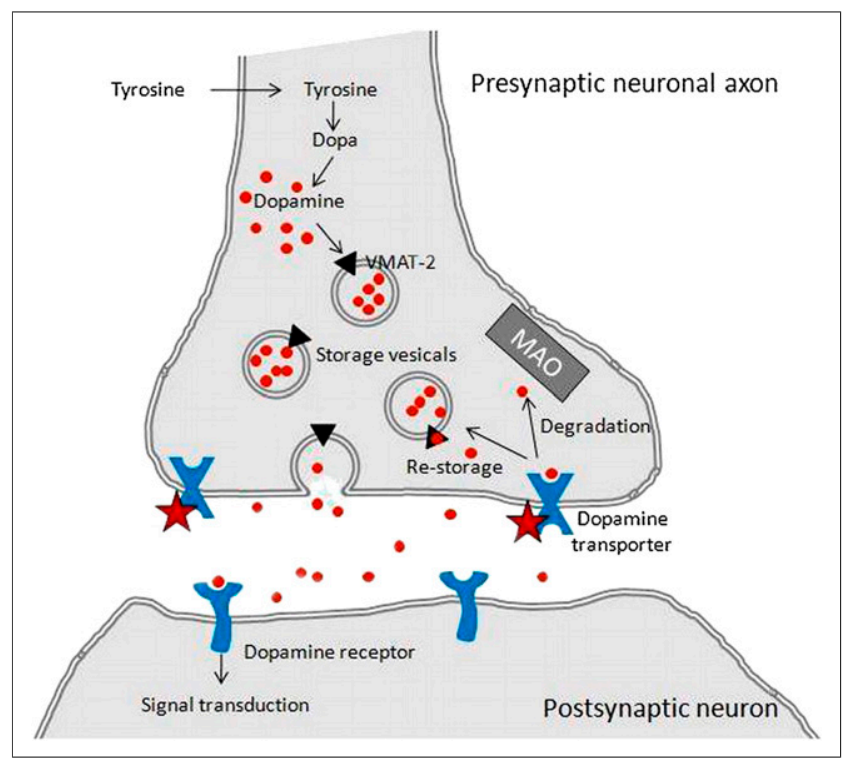

FIGURE 2. Dopamine transporter synaptic cleft. Stars represent where ${ }^{123}$-ioflupane binds to transporters. (This image was originally published in (9).) solution. After the thyroid-blocking agent is given, there is an hour wait before the intravenous tracer injection. The adult dose is generally $185 \mathrm{MBq}(5 \mathrm{mCi})$, with a range of 111-185 $\mathrm{MBq}$ (3-5 mCi) (9). Imaging occurs 3-6 h after tracer injection to allow the maximum uptake into the dopamine transporters. After the 3- to 6-h period, the patient is imaged with SPECT on a multihead nuclear medicine $\gamma$-camera. Images of the patient's entire brain are taken over a 35- to 40-min scan time, with proper head positioning. Accurate head placement will provide precise visualization of the basal ganglia and specific regions involved such as the caudate nucleus and putamen. Head placement can be achieved with positioning devices such as a head cradle, straps, and cushions.

Minor variations in the protocol are allowable and are usually site-specific, however, this outline serves as a general guideline to what is typically performed. Detailed image acquisition techniques may be found in the SNMMI Practice Guideline for Dopamine Transporter Imaging with ${ }^{123}$ I-Ioflupane SPECT 1.0 (9).

After images are obtained, the processing steps begin with a sinogram of the data as well as motion correction and image reformatting into coronal, sagittal, and transaxial slices. The slices for diagnostic interpretation are chosen on the basis of a visual selection of the most intense tracer uptake. The slices of the transaxial plane with the highest striatal activity in the basal ganglia are used in a process known as semiquantification. The SNMMI protocol defines this as "the ratio of activity in a structure of interest to activity in a reference region" (9).

For semiquantification of ${ }^{123} \mathrm{I}$-ioflupane DaT SPECT, binding ratios are calculated by comparing activity in the striatum with activity in an area of low DaT concentration (usually the occipital area) (9). To obtain these ratios, regions of interest (ROIs) for the putamen and caudate nucleus of each hemisphere need to be designated. There are 4 ROI methods: manual ROIs, manual volumes of interest (VOIs), more advanced automated systems using VOIs, and voxel-based mathematic systems (9). The manual ROI method is most often used and will be discussed as the principal method when processing the image slices in this article. However, this technique usually leads to the highest instance of interobserver variability, which may translate into an inaccurate interpretation of the data.

In visual interpretation, the striatal shape, extent, symmetry, and intensity differentiate normal from abnormal. The normal striata on transaxial images should look crescent or comma-shaped and should have symmetric well-delineated borders. Abnormal striata will have reduced intensity on one or both sides, often shrinking to a circular or oval shape (9).

These observations of normal versus abnormal help delineate essential tremor from idiopathic Parkinson disease and the other parkinsonian syndromes. In the final interpretation, those with normal uptake images are assumed to have essential tremor, whereas any abnormalities will be deemed Parkinson disease or the other parkinsonian 
syndromes. In conjunction with the semiquantification analysis from the ROI selections, the visually ascertained results often present a general estimation of tracer uptake in the images. Despite these results, there is the ongoing issue of interobserver variability in ROI selection as well as the lack of an established normals database to compare with individual scan results. The ROI selection methods, varying camera models in use, and processing tool variations all contribute to the lack of a recognized standard of image interpretation for the DaT scan protocol. Here the GE Healthcare software program known as DaTQUANT is recognized as a key processing component to remedy these issues at hand. Other helpful components of the software will be discussed as additional features to promote widespread use.

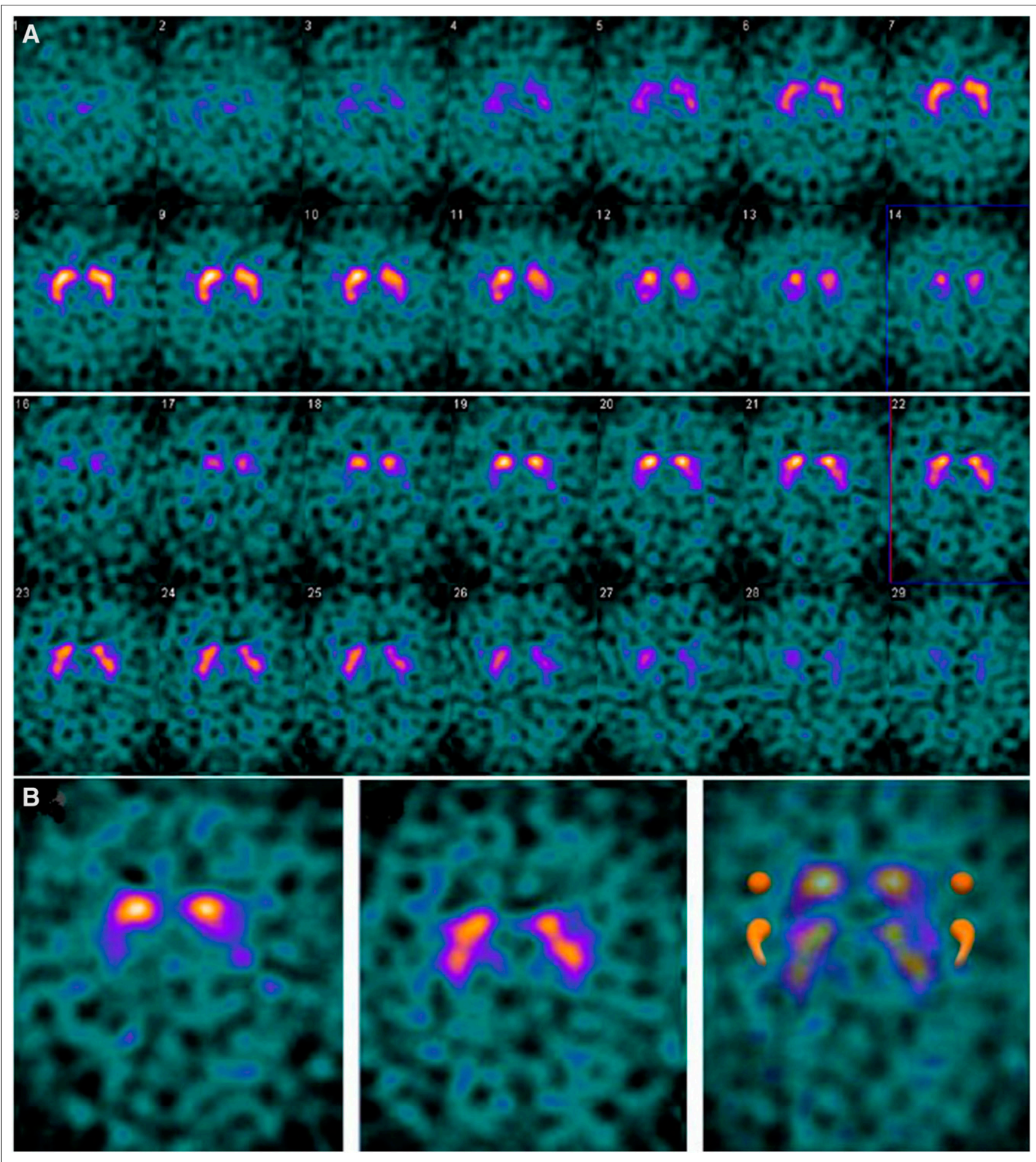

FIGURE 3. (A) DaT scan images visualizing "semicolon sign." (B) Forward head-tilt artifact, which falsely represents caudate nuclei separate from putamen. False-positive artifact. (This image was originally published in (11).) 


\section{DATQUANT}

Launched in 2013 by GE Healthcare, DaTQUANT is available for use with any GE Healthcare DaT scan protocol and approved SPECT camera. According to GE Healthcare (2018): "an aid in the assessment of the degree of in-vivo expression of the dopamine transporter (DaT), DaTQUANT assists in the detection and quantification of the loss of functional striatal dopaminergic neuron terminals, a loss correlated with Parkinson disease. Quantitative analysis may help assess the extent and intensity of the striatal ${ }^{123}$ I Ioflupane signal" (10).

Instead of manually selected ROIs, the software uses a predefined voxel of interest (VOI) template for automatic asymmetry measurements and putamen-to-caudate uptake ratios. Here the degree of variation in the regions of uptake can be quantified in a volumetric measurement, including the ratio of uptake between the distinct portions. The software is over $95 \%$ accurate in automatic VOI placement, with the option of manual adjustments as needed (10). DaTQUANT eliminates the need for manual ROI selection and potentially eliminates interobserver variability with programmed VOI selections.

A critical issue within the DaT scan is the head-tilt artifact that can occur when positioning patients. Per protocol, the patient lies supine on the imaging table with their head placed in a supportive device to prevent any cranial movement or tilt. Any involuntary tremors during the scan or improper securing can lead to the head-tilt artifact (Fig. 3) . DaTQUANT can redirect any image automatically back to the optimal orientation of the anatomy within the images, which can remove the head-tilt artifact as well as the potential problem of inaccurate VOI placement.

The software reports the putamen-to-caudate ratios and left-to-right asymmetry ratios. The software analysis can determine the remaining presence of functional striatal dopaminergic neuron terminals for the differentiation of essential tremor from Parkinson disease. Other values provided when using DaTQUANT include a calculated mean of uptake, a specific deviation of uptake expressed as a percentage (SD of 1), and a VOI thickness (known as $z$ score) value. These values are based on a normals database from the Parkinson Progression Markers Initiative, a comprehensive study of patients with diagnosed Parkinson disease (10). In using the data from this study, GE Healthcare was able to generate an age-matched normals database across a spectrum of ages, providing each individual case a nearly exact representation of their results in comparison to their counterpart from the database (Fig. 4).

In the latest iteration, the software provides longitudinal image analysis to aid in the monitoring of disease advancement and enables access to both a visual and a quantitative comparison of a patient's images over several scans. Patients who require multiple scans due to symptom changes

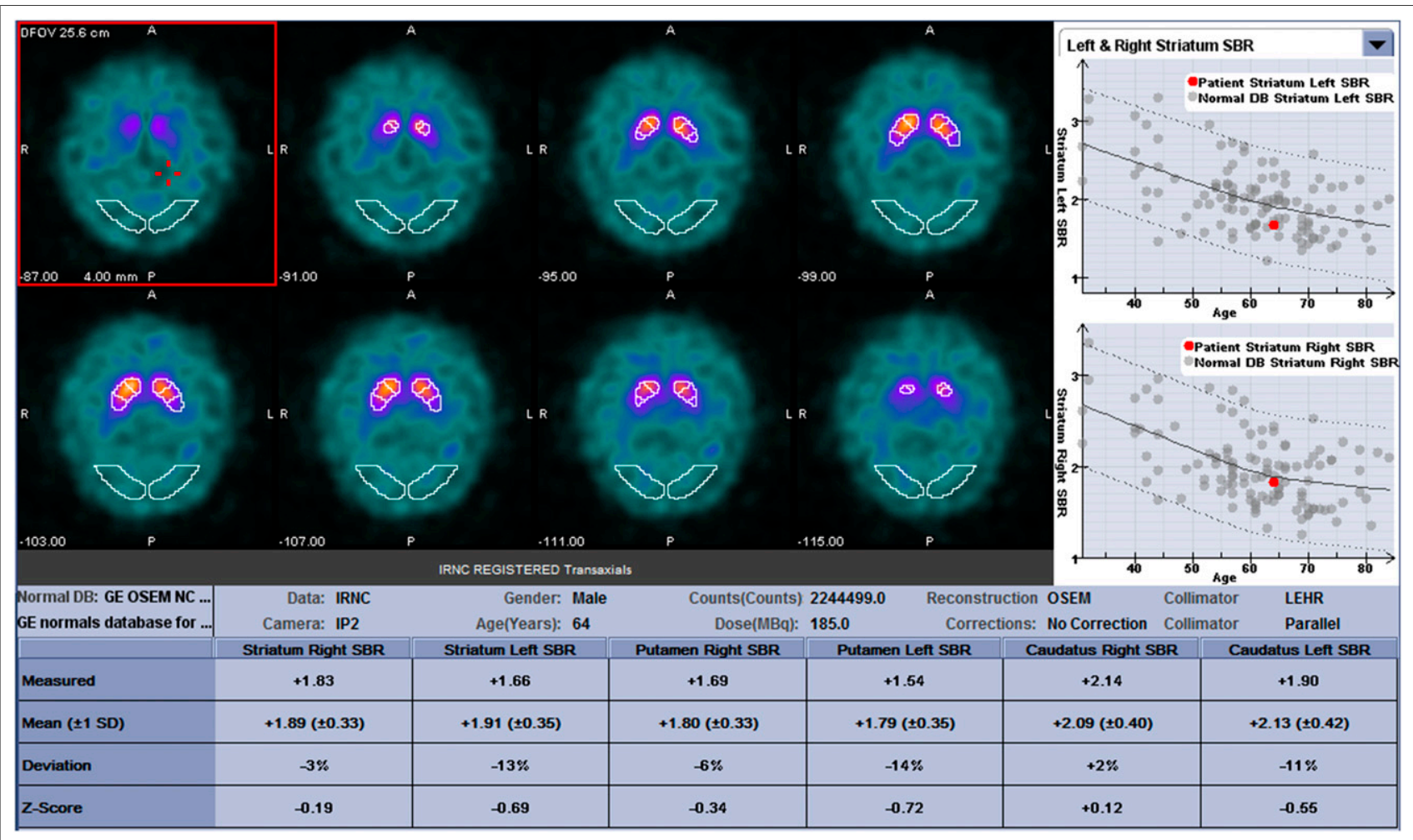

FIGURE 4. Sample save screen of the DaTQUANT (GE 2018) processing: white VOI placement in slices of images; normals database on top right, with red dots representing individual case results against range of normal database cases. Other calculated data at bottom of image. (This image was originally published in (12).) 
or medication issues can benefit from this capability. In terms of clinical data, a dopaminergic transporter density report is available for interpreting physicians to review. GE Healthcare offers the software to any site using the ${ }^{123} \mathrm{I}-$ ioflupane protocol with approved SPECT cameras. Despite limited use in the last $5 \mathrm{y}$, this emerging technology holds the key to Parkinson disease evaluations with a better understanding of the patient's condition at the striatal level. Providing conclusive answers to those experiencing parkinsonian syndromes means one step closer to a final diagnosis and better symptom management.

\section{CONCLUSION}

In the differentiation of Parkinson disease from essential tremor, the DaT scan protocol using DaTQUANT provides a patient with an accurate in vivo evaluation of brain physiology. The current diagnostic practices have been improved with this modality, leading to better symptom management and treatment. The latest enhancements of the software continue to expand the capabilities of the protocol, providing a patient with more answers regarding their results. Overall, the GE Healthcare ${ }^{123}$ I-ioflupane protocol with DaTQUANT has led to many advancements with more diagnostic information, which someday may help in discovering a cure.

\section{DISCLOSURE}

The Brain and Body Donation Program is supported by the National Institute of Neurological Disorders and Stroke (U24 NS072026 National Brain and Tissue Resource for Parkinson's Disease and Related Disorders), the National Institute on Aging (P30 AG19610 Arizona Alzheimer's Disease Core Center), the Arizona Department of Health Services (contract 211002, Arizona Alzheimer's Research Center), the Arizona Biomedical Research Commission (contracts 4001, 0011, 05-901, and 1001 to the Arizona Parkinson's Disease Consortium), and the Michael J. Fox Foundation for Parkinson's Research. No other potential conflict of interest relevant to this article was reported.

\section{ACKNOWLEDGMENTS}

I acknowledge and thank Lisa Leigh Patrick and Joyce Zimmerman for their mentorship and consideration in publishing this article. I also thank the Gila River Indian Nation for their continued support of my educational journey. We are grateful to the Banner Sun Health Research Institute Brain and Body Donation Program of Sun City, Arizona for the provision of human brain tissue.

\section{REFERENCES}

1. Statistics on Parkinson's disease. Parkinson Association of the Carolinas website. https://www.parkinsonassociation.org/facts-about-parkinsons-disease/. Accessed January 24, 2019.

2. Madame Curie Bioscience Database [Internet]. Dopamine and Parkinson's disease. National Center for Biotechnology Information, U.S. National Library of Medicine website. https://www.ncbi.nlm.nih.gov/books/NBK6271/. Accessed January 24, 2019.

3. Parkinson's disease. Mayo Clinic website. https://www.mayoclinic.org/diseases conditions/parkinsons-disease/diagnosistreatment/drc-20376062. Accessed January 24, 2019.

4. Parkinson's disease medications. Parkinson's Disease Clinic and Research Center website. http://pdcenter.neurology.ucsf.edu/patients-guide/parkinson's-disease-medications. Accessed January 24, 2019.

5. Ba F, Martin WR. Dopamine transporter imaging as a diagnostic tool for parkinsonism and related disorders in clinical practice. Parkinsonism Relat Disord. 2015;21:87-94.

6. Mandal A. Parkinson's disease history. News-Medical.Net website. https://www. news medical.net/health/Parkinsons-Disease-History.aspx. Updated August 23, 2018. Accessed January 24, 2019.

7. Goetz CG, Tilley BC, Shaftman SR, et al. Movement Disorder Society-sponsored revision of the Unified Parkinson's Disease Rating Scale (MDS-UPDRS): scale presentation and clinimetric testing results. Mov Disord. 2008;23:2129-2170.

8. GE announces FDA approval of DaTscan. Parkinson's Progression Markers Initiative website. https://www.ppmi-info.org/2011/01/ge-announces-fda-approval-of-datscan/. January 14, 2011. Accessed January 24, 2019.

9. Djang DS, Janssen MJ, Bohnen N, et al. SNM practice guideline for dopamine transporter imaging with ${ }^{123} \mathrm{I}$-ioflupane SPECT 1.0. J Nucl Med. 2012;53:154163.

10. DaTQUANT ${ }^{\mathrm{TM}}$. GE Healthcare website. https://www.gehealthcare.com/products/nuclear imaging-agents/datquant. Accessed January 24, 2019.

11. Covington MF, McMillan NA, Avery RJ, Kuo PH. The semicolon sign: dopamine transporter imaging artifact from head tilt. J Nucl Med Technol. 2013;41: $105-107$.

12. Booij J, Dubroff J, Pryma D, et al. Diagnostic performance of the visual reading of ${ }^{123}$ I-IoflUPANE SPECT images with or without quantification in patients with movement disorders or dementia. J Nucl Med. 2017;58:1821-1826. 